\title{
L'épreuve de la voix : la scène primitive de la représentation
}

Voice Test: The Primal Scene of Representation

\section{Helga Finter}

\section{(2) OpenEdition}

Journals

Édition électronique

URL : https://journals.openedition.org/rsl/1329

DOI : $10.4000 /$ rsl. 1329

ISSN : 2271-6246

Éditeur

Éditions Rue d'Ulm

Référence électronique

Helga Finter, «L'épreuve de la voix : la scène primitive de la représentation », Revue Sciences/Lettres [En ligne], 6 | 2019, mis en ligne le 10 décembre 2018, consulté le 30 juillet 2021. URL : http:// journals.openedition.org/rsl/1329; DOI : https://doi.org/10.4000/rsl.1329

Ce document a été généré automatiquement le 30 juillet 2021.

(C) Revue Sciences/Lettres 


\section{L'épreuve de la voix : la scène primitive de la représentation}

Voice Test: The Primal Scene of Representation

Helga Finter

\section{Observation (1) : du rythme textuel}

Dans le programme strasbourgeois pour sa mise en scène du Temps et la chambre de Botho Strauss ${ }^{1}$, Alain Françon rapporte une observation qu'il avait faite pendant la préparation du spectacle. Elle concernait l'établissement de la relation entre texte et voix : le metteur en scène avait fait lire à haute voix le texte français de la pièce par son traducteur, Michel Vinaver, arguant que lui seul savait, en tant qu'écrivain, «poser les intensités là où il faut ». Cette lecture avait servi, d'une part, à repérer le tempo du débit vocal - «staccato ou fluide" - et les pauses indiquant ce qui devait être souligné, d'autre part, à définir les accents toniques qui, bien que potentiellement libres dans la langue française, se font distinctifs en précisant une pensée ou un sens. Le travail des répétitions consistait alors à déterminer avec les acteurs tous ces traits du rythme de la phrase avant de répéter sur scène. Selon Alain Françon, cette investigation du rythme du texte dramatique fait que les personnages ou figures se précisent et peuvent advenir sur scène, en dehors de toute recherche psychologique.

2 L'observation du metteur en scène implique l'hypothèse suivante : si ce sont le rythme et l'accent tonique qui créent la représentation du personnage, cela veut dire que c'est la voix ou les voix du texte que l'acteur aura à dégager et à entendre avec son oreille intérieure afin de la (les) rendre audible(s) comme voix d'un autre/de l'autre dans sa propre voix. C'est par le vocal, la vocalité, que la représentation sera rendue présente. À l'origine de la représentation il y a d'abord une épreuve de la voix.

3 Alain Françon n'est pas le seul metteur en scène à placer au centre du travail théâtral l'exploration du rythme et de la voix du texte. Ce souci distinguait aussi, avant 1989, l'acteur de l'Allemagne de l'Est, formé, selon les préceptes brechtiens, au travail du texte, de celui de l'Ouest, adepte d'une introspection psychologique à la Stanislavski ou 
à la Strasberg. Cette importance du travail sur le rythme textuel se trouvait également chez des metteurs en scène aussi différents que Hans Jürgen Syberberg, Jean-Marie Straub et Danièle Huillet ou Klaus Michael Grüber. En Italie, ce fut le souci majeur aussi bien de Carmelo Bene que de Luca Ronconi qui affirmait, comme Françon, que l'identification du rythme et de la voix du texte faisait automatiquement suivre le geste $^{2}$. En France, on trouvait des adeptes de cette conception de la voix du texte, parmi les metteurs en scène - comme Claude Régy et Patrice Chéreau -, mais surtout parmi les dramaturges tels que Marguerite Duras, Eugène Ionesco, Jean Genet, Samuel Beckett ou Valère Novarina. Comment le rythme de la parole textuelle peut-il créer le personnage et entraîner la figuration gestuelle de son corps?

\section{Observation (2) : technologies sonores sur scène}

4 Avant de répondre à cette question, une seconde observation s'impose : la mise en scène du Temps et la chambre fut l'une des rares, dans la période récente, à n'avoir recours ni à l'amplification de la voix par un ou des micros ni à une sono étourdissante. Et quand elle faisait entendre une voix acousmatique, cette décision suivait la logique dramatique du texte qui la décrivait comme une voix venant d'une "colonne située dans la chambre ». Les différents personnages ou figures qui évoluaient sur cette scène et y changeaient d'emploi avec une légèreté kaléidoscopique étaient les effets de paroles écrites, émises à haute voix, voix présentant la parole poétique comme performative de son propre effet de représentation. Celui-ci était-il dû, comme il le sembla de prime abord, à l'absence de technologie sonore?

5 J'ai présenté ailleurs ${ }^{3}$ une réflexion sur les gains et pertes entraînés par l'emploi de technologies sonores sur scène, en considérant ces dernières sous deux aspects. Elles apparaissent d'abord comme une prothèse ou orthèse suppléant les déficits acoustiques de la salle, les déficits d'audition du public et/ou les insuffisances vocales des acteurs en n'obéissant qu'à des critères d'efficacité. À cet emploi purement instrumental des technologies sonores s'oppose un second emploi, qui articule leur usage à un projet esthétique d'interrogation de la fonction de la voix dans son rapport à la parole, comme ce fut le cas chez Robert Wilson, Richard Foreman, Samuel Beckett, Carmelo Bene, et comme ça l'est encore chez Romeo Castellucci, Heiner Goebbels, Krysztof Warlikowski, Joris Lacoste, entre autres.

6 Je souhaite ici rappeler quelques-uns des gains et pertes liés à l'emploi des technologies sonores. Du côté positif, se rangent l'audibilité de la parole de l'acteur jusqu'au dernier rang de n'importe quelle salle; le close up auditif projetant l'acteur, mais aussi l'auditeur, dans l'intimité de la voix entendue malgré l'éloignement physique de l'acteur; la séparation de la voix et du corps présent sur scène, déconstruisant le fantasme d'un corps vocal originaire soudé à un corps physique, mais créant aussi des signifiants auditifs de l'atopie transcendantale de la voix, comme le halo ou la réverbération chez Carmelo Bene ; la capacité de la technologie sonore à transformer et à modifier les voix d'une façon dont l'acteur ne serait pas capable. Du côté négatif, il faut noter l'unification et l'égalisation du son, l'effacement des différences vocales et l'intégration à une ambiance sonore de base, déterminée par le sound designer, qui dépersonnalise le débit singulier en lui enlevant son timbre spécifique. Or l'amplification vocale peut en effet procurer à l'acteur la jouissance narcissique de son miroir acoustique tout en interpellant en même temps celle de l'auditeur. Cette 
amplification sonore fictionnalise cependant aussi, pour le spectateur-auditeur, la présence scénique : le mirage d'un son unifié et séparé du corps prive le spectateur de la confrontation avec la parole d'un autre, avec une parole autre. Le dialogue scène-salle y est remplacé par un soliloque imposé et narcissiquement autosuffisant. En même temps, cette illusion narcissique se double de celle du spectateur : celui-ci se miroite dans une parole qui bien que sortie du haut-parleur peut lui sembler être intimement adressée, dite à son oreille. L'invocation de cette voix amplifiée a alors un effet analogue à celui qu'exerce la voix du ténor Edgar Lagardy sur Madame Bovary quand elle se sent personnellement interpellée par le chanteur sur scène lors d'une représentation de Lucia di Lammermoor à l'Opéra de Rouen4.

7 Le théâtre s'approche alors de l'illusion imaginaire du cinéma : l'effet du tout sonore technologique soumet le spectateur à un " acousmêtre $^{5}$ » brouillant la source des voix. Les voix acousmatiques patentes soulignent cet effet : la scène devient récit d'un " je » caché, comme c'est le cas dans beaucoup de spectacles de danse, où les danseurs semblent des marionnettes présentées par la voix d'un maître invisible au fond de la scène, dans les cintres ou les haut-parleurs, scène surgie de la voix du maître (his master's voice), omniprésent, omnipotent, omniscient, tant qu'il ne se désacousmatise pas, comme par exemple chez Pippo Delbono ou Emma Dante.

8 Dans ce théâtre devenu cinéma, récit ou emblème, voire théâtre de marionnettes, la voix de la parole est noyée dans les images, les bruits et les sons, tout en étant égalisée dans ses registres par l'enregistrement: elle est empreinte du style du créateur son tout en étant noyée dans un tapis de son environnemental. La souveraineté de cette parole n'est plus portée par la voix même ou par celui qui l'émet. Elle est le fait du metteur en scène, maître de la machinerie et seigneur caché du spectacle, qui se présente à travers les voix manipulées.

9 L'engouement pour l'opéra, seul lieu où la technologie sonore semble encore bannie à part quelques exceptions marquantes ${ }^{6}$, me paraît alors significatif, surtout ces dernières années et chez un public plus jeune, d'une fatigue des voix formatées et uniformisées. Il en va de même pour les recherches théâtrales qui intègrent le chant comme celles de François Tanguy, Christoph Marthaler, Sylvain Creuzevault, entre autres. Que nous dit cette propension à la voix chantée en dehors de l'addiction du mélomane?

\section{Voix souveraines (1) : le chant}

À l'opéra, la voix amplifiée ou augmentée est interdite et le soupçon de soutien technologique équivaut à un soupçon de fraude. L'enjeu de la voix du chanteur d'opéra, mais aussi du chanteur de lied, est lié à ce que représente le chant comme capital symbolique, dans une société qui l'enferme dans une institution propre: la place réservée au chant est, d'une part, celle, hétérotopique, de la voix-jouissance du corps autrement, rivée à la parole et absorbée par elle ${ }^{7}$. D'autre part, le chant est aussi et surtout l'endroit où se célèbre l'immanence de sa transcendance: la voix du chanteur d'opéra tient son pouvoir d'un effet de souveraineté dont témoigne encore le surnom de la diva, la divine, comme celui du divo, le divin. L'opéra est censé manifester ce qui permet la parole, la rend effective, une légitimité qui lui vient du souverain, et à travers lui, en dernière instance, de dieu: jusqu'au xviII ${ }^{e}$ siècle, les voix de dessus indistinctement d'hommes ou de femmes - étaient appelées soprano et censées 
représenter une puissance légitimée par le souverain et léguée par une instance transcendantale ${ }^{8}$.

11 Avec la mise à mort du roi, puis celle de dieu, cette souveraineté s'est clivée en au moins trois instances : celle portée par les chœurs qui parlent pour le peuple souverain, de plus en plus présents dans l'opéra du XIX ${ }^{e}$ siècle ; celle portée par la voix soprano de femmes promettant la jouissance d'un paradis perdu payée souvent par leur propre mort, la voix de l'Éternel féminin ; celle portée par la basse ou le baryton basse, garante de la Loi, que nous trouvons depuis Bellini et Verdi. Cette concurrence d'instances - le Peuple, l'Éternel féminin et la Loi -, légitimant la voix du chant, mélange les horizons du politique, du social et du familial qui dès lors ont usurpé la place du religieux. Au $\mathrm{xx}^{\mathrm{e}}$ siècle, cette tripartition cède la place à un tissu de voix singulières et plurielles, dont témoignent les opéras depuis Strauss, Schoenberg, Berg et Janaček jusqu'à Nono, Berio, Reich et Dusapin par exemple. Avec eux l'horizon du chant se dégage du mythe politique et familial. La langue seule et le langage, le symbolique du grand Autre, seront l'horizon transcendantal de la voix et du chant.

\section{Voix souveraines (2) : la parole légitime}

Le théâtre semble très éloigné de devoir légitimer une vocalité affichée. Ne tient-il pas souvent sa raison d'être de son parler «naturel », évoluant au fil du temps : aujourd'hui celui du cinéma, ou le parler familier voire populaire, celui des classes dites "populaires»? Cet état de la langue théâtrale, comme l'amplification cinématographique voire médiatique de la scène, cache la spécificité de la voix de la parole au théâtre.

Il faut ici, d'une part, insister sur le lien existant entre religion et théâtre, rappelé par Bertolt Brecht dans son «Petit Organon » : « Dire que le théâtre vient du culte, signifie seulement que le théâtre ne devenait théâtre que par le fait d'en sortir ${ }^{9}$ ». Il faut, d'autre part, se souvenir du lien unissant l'opéra à la tragédie antique : l'invention de ce nouveau genre dans la Florence de 1600 par la Camerata de' Bardi, avec Jacopo Peri, Giulio Caccini, entre autres, se comprenait justement comme une réinvention du théâtre grec dont on voulait réactualiser la déclamation-mélopée. Sans oublier qu'à la même époque, d'abord à Mantoue, puis à Venise, l'opéra de Claudio Monteverdi visait avec sa seconda pratica un primat de la parole sur la musique. Ainsi, l'énonciation vocale sur scène implique un dispositif pour la voix trahissant un double héritage, celui du rite religieux et celui du théâtre antique.

Pour reconstituer ce qu'a été le commencement de notre théâtre, à la Renaissance italienne, on doit tenir compte du double passé qui présidait à sa genèse : il devait tout à la fois s'échapper du Mystère sacré et s'affirmer face au théâtre antique. Ceci impliquait non seulement qu'il parlât une langue compréhensible par tous, la langue vernaculaire - volgare -, délaissant et la langue de la Bible et celle des Anciens, et qu'il ne traitât plus exclusivement de sujets bibliques, ou venus de l'érudition antique, il fallait aussi qu'il se donnât un dispositif légitimant une prise de parole théâtrale. Ce dispositif lui venait non du théâtre antique mais de la transformation de formes scéniques qui avaient autrefois fondé un mode sacré de représentation.

Pour le Mystère déjà, l'abandon du latin avait nécessité une justification transcendantale fournie dans le début du drame sacré: annuncio ou annunzio ${ }^{10}$. Il 
s'agissait de l'annonciation de l'action sacrée, chantée par un ange, lui-même introduit par le son d'un instrument de cuivre ou de percussion qui, au début de la représentation, avait préparé le public au mystère du jeu d'une présence de l'absence. Celui-ci en effet a d'abord été présenté, depuis le $\mathrm{x}^{\mathrm{e}}$ siècle, comme jeu autour du tombeau vide du Christ - visitatio sepulchri - puis élargi à l'Annonciation à la Vierge ${ }^{11}$, de même qu'à la visite des rois mages - Jeux de mages ${ }^{12}$.

Cette annonce se transforme en prologue dans le théâtre de cour de la Renaissance italienne, ancêtre du théâtre de l'ère moderne. Le Prologo de l'auteur présenté par luimême ou représenté par un acteur ouvre la représentation. Il est souvent suivi par un second prologue, celui de l'allégorie du texte - Testo - proposant soit le résumé de l'action, soit l'allégorie du genre dramatique - Tragedia ou Commedia - justifiant, comme le prologue de l'auteur, le langage et le style choisis ${ }^{13}$.

Toute la mise en scène de ce commencement rend cependant compte des traces sacrées de cette annonce laïque : à force de trombes ${ }^{14}$ et de tambours ainsi que de tombées de rideaux, le prologue du théâtre italien de la Renaissance emprunte à l'Annonciation chrétienne les signifiants présageant une révélation sacrée. C'est alors la présentation de son discours même qui rendra légitime la prise de la parole au théâtre : bien que la parole sur scène ne soit plus sacrée, elle sera justifiée par une altérité transcendante caractérisant l'âme du poète, son inventio et sa furor ; ces catégories néoplatoniciennes émanant du primo amor divin légitiment une voix singulière de poète comme signature divine ${ }^{15}$. Cette parole autre peut alors englober, dans le cas de la comédie, la totalité humaine dans ses contrastes entre styles haut et bas. Ainsi Ludovico Ariosto explique-til, par exemple, les mots d'esprit érotiques de sa comédie La Cassaria dans le prologue par le plaisir esthétique - «pour rendre la fable moins triste ${ }^{16} »-$ afin de justifier une licence en contraste avec les préceptes de conversation prônés par la bienséance en vigueur à la cour.

Il y a donc au théâtre aussi une parole spécifique. Elle est celle d'un auteur, d'un poète. Elle n'absorbe pas la voix, au contraire. C'est la voix exhibée qui rend cette parole présente. Elle se fait entendre grâce à une voix qui, étant le médium d'un poète, la porte et la légitime. Leone de' Sommi a décrit le dispositif de la présentation de cette parole au milieu du Cinquecento ( $\mathrm{xvI}^{\mathrm{e}}$ siècle) : elle est dite face au public à partir du milieu du proscenium, son porteur se faisant point de fuite pour le regard du spectateur. Une musique acousmatique peut la soutenir, provenant du fond de la scène en perspective où la source est cachée (comme au Teatro Olimpico de Vicence), émise des deux tours situées des deux côtés de la scène (comme pour La Calandria à Urbino), ou du fond de la salle, du haut d'un balcon, à l'arrière du public (comme au théâtre des Médicis aux offices de Florence). Le second prologue, le résumé de l'action par le personnage du Testo, est lui aussi emprunté au Mystère mais va bientôt disparaître, et déjà chez l'Arioste lui-même. C'est alors au spectateur de deviner la trame de l'action ou de recréer le personnage, un personnage pour lequel, quand il s'agit d'une comédie nouvelle, il ne dispose d'aucun savoir antérieur.

Comment le spectateur s'y retrouve-t-il ? Le personnage se présente par une parole présentant la représentation. Parole préfigurée dans les répliques du texte dramatique qui propose des voix et aussi - par le style de son écriture et par sa versification - la voix du texte.

20 C'est l'écriture du poète, le style ou le vers du texte, qui légitime la voix sur scène. Ce n'est pas le caractère vraisemblable de ce parler qui décide de sa légitimité, mais sa 
qualité d'écriture, laquelle lui confère sa souveraineté par l'invocation de la langue comme a/Autre.

21 La voix sur scène intervient alors comme venant d'un ailleurs : du poète qui est dit « inspiré » en effet. C'est ce qui identifie cette voix autre, son « rythme», qui légitime l'acteur et lui transfère sa souveraineté. Ainsi la voix peut-elle rendre présent le personnage tout en présentant la représentation.

\section{Scènes primitives de la représentation}

L'œuvre de la voix rappelle une double scène originaire : la voix permet de dire «je » et, ce faisant, permet de se présenter comme sujet parlant. Elle crée le sujet dans la langue: «Je dis donc je suis». Mais cet acte de dire présente en même temps la représentation par la parole comme rendue possible par la voix. C'est justement cette deixis vide de l'appareil linguistique du sujet qu'Émile Benveniste ${ }^{17}$ avait mise au jour en montrant comment le pronom qui permet à chacun, chacune de se dire, le/la soumet en même temps au langage. C'est la voix qui module cette soumission tout en la présentant comme représentation d'un « je ». La voix exhibée sur scène présente donc toujours aussi l'origine de la représentation, au-delà du miroir narcissique.

Ce premier acte de langage en rappelle un second : l'acte eucharistique réalisant dans le rite catholique la présence réelle du Christ. Cet acte est un acte de parole par lequel le prêtre répète celui du Christ à la dernière Cène - "Ceci est mon corps [hic enim est corpus meum], ceci est mon sang [hic enim est calix sanguinis mei] ». La deixis " ceci/hic » indique en premier lieu l'hostie et le vin blanc ${ }^{18}$. C'est l'actualisation vocale des mots du Christ qui opère, pour le croyant, leur transsubstantiation en chair et en sang. Elle est à la fois symbolique, imaginaire et réelle: le vin et l'hostie sont des symboles et des images du sang et $d u$ corps $d u$ Christ et, en même temps, ils sont la présence réelle de ce sang et de ce corps.

\section{Actes de parole}

La voix est d'habitude le refoulé de la parole tout en la rendant possible. En ceci elle est la mort à la base du langage comme l'a analysé Giorgio Agamben ${ }^{19}$. L'acte de parole, d'une parole performative rendue effective par la voix, produit un présent qui est à la fois réel, symbolique, et imaginaire. Mais, ce faisant, la voix qui porte cette parole doit d'abord être légitimée par un cadre ou un pacte symbolique comme c'est le cas pour celle de l'officiant du rite.

Ainsi la voix qui d'habitude est le refoulé de la parole doit au théâtre se faire entendre comme l'instance qui fait advenir la parole, et donc vient d'un ailleurs. Ce dont il s'agit alors est l'exhibition d'une relation vocale triple : à la fois au texte, à la langue et au corps de l'acteur.

Nous connaissons tous la fameuse scène du Midsummer Night's Dream de Shakespeare où une troupe d'artisans réunie autour de Bottom tente de créer de toutes pièces par ses paroles la tragédie de Pyrame et Thisbé. Prêtant à rire par leurs voix - ton, timbre et mélos -, ces acteurs amateurs s'avèrent inadéquats, car incapables de s'abstraire de leur propre voix, voire d'habiter la parole qui fait d'eux des sujets autres. Les mêmes travers affectent les voix des valets campagnards dans Les Acteurs de bonne foi de 
Marivaux: incapables eux aussi de se détacher de leur parler paysan et d'habiter vocalement et mentalement le texte d'un autre.

Tel est pourtant le défi de la scène théâtrale. Le personnage doit être présent par les premières paroles énoncées: Ubu Roi avec l'interjection «Merdre!», Estragon avec "Rien à faire", suivi par Vladimir avec «Je commence à le croire ». Clov s'adresse au public avec "Fini, c'est fini, ça va finir. Ça va peut-être finir », Krapp lâche «Ah! Boîte... trrrois... bobine... cinq ", Winnie exulte : "Encore une journée divine » avant une prière ânonnée et le rituel de la brosse à dents. Du fantoche de Jarry, les personnages de Samuel Beckett se distinguent cependant par le mode d'entrée en scène de cette parole : tandis que l'explosion des consonnes agglutinées du « Merdre » d'Ubu annonce meurtres, terreur et guerres grotesques, les paroles des personnages de Beckett se présentent comme issues d'un processus de différenciation d'avec des bruits corporels indistincts. Les paroles d'Estragon sont précédées de l'«ahanant» et du « haletant » de gestes corporels. L'adresse de Clov surgit d'une suite de quatre « rires brefs » accompagnant des actions physiques répétées. Krapp sort sa première parole après un va-et-vient bruyant, des soupirs et l'absorption bruyante de bananes. Et les premières paroles de Winnie émergent des sonneries répétées d'un réveil. La voix qui charrie la parole se détache alors de bruits organiques, de souffles et de halètements, d'une part, de bruits mécaniques, d'autre part.

La légitimation de la parole sur scène se fait ici par une exhibition de la voix du personnage mise en relation avec son corps ou avec une mécanique étrangère. L'insistance sur le corps et le souffle indique sur quoi repose l'acte de parler: sur le refoulement de la voix, le refoulement de la mort. Cette dernière se maintient simultanément par la composition rythmique qui l'articule et la sublime comme une basse continue.

Une voix d'acteur sans artifices technologiques peut alors, en nous donnant le plaisir rassurant d'une présentation de la parole, rejouer l'origine de la représentation du sujet, grâce à l'art vocal de l'acteur : se présenter avec la voix d'un autre - la voix de la langue - pour se représenter.

\section{Épilogue : nouvelles voix souveraines (3)}

L'artifice de l'amplification et de la sonorisation, quand il n'est plus purement instrumental, nous indique, de surcroît, les enjeux d'une nouvelle instance de souveraineté vocale issue cette fois de la société du spectacle. En effet, disposer du micro légitime une parole, la technologie sonore lui confère du pouvoir. Ainsi, l'emploi de micros peut s'imposer pour un travail sur la voix politique : qui s'empare du micro aura la parole, comme dans Ça ira (1). Fin de Louis de Joël Pommerat ${ }^{20}$. De même l'exploration des voix d'aujourd'hui où, même dans le quotidien, celles qui ne sont pas assistées semblent minoritaires, nous fournit tout un florilège de productions vocales enregistrées et médiatisées, celle du téléphone, de skype, de la télévision, de la vidéo, de l'évènement sportif, etc. Ce sont de telles voix qu'a fait entendre Joris Lacoste dans la Suite $n^{\circ} 2$ de l'Encyclopédie de la parole ${ }^{21}$ : la reproduction en chœur de paroles dites avec - et transmises par - une prothèse ou orthèse sonore souligne, au-delà de l'appareillage sonore, un autre horizon transcendantal - le rôle légitimant du rythme (intonation, accents, tempo) de ces paroles; les médias peuvent favoriser la concentration sur ce rythme sans entraver l'audibilité des paroles. Ce sont les voix de 
paroles qui créent la jouissance à partir des voix de la langue. Comme dans la musique d'un Luciano Berio, d'un Steve Reich ou d'un Robert Ashley. Ces compositeurs avaient prévu pour leurs œuvres vocales l'assistance de technologies sonores, mais ils créent, par contre, leur chant à partir du mélos de paroles singulières, mettant en avant la prosodie et des sons des langues respectives. En invoquant les voix de la langue comme un ailleurs, les voix des paroles confèrent une présence éphémère à une multitude de personnages dont elles présentent vocalement la représentation.

\section{NOTES}

1. Alain Françon, «Entretien » (avec Fanny Mentré, le $1^{\mathrm{er}}$ avril 2016, Paris), programme du spectacle Le Temps et la chambre de Botho Strauss, Théâtre national de Strasbourg, novembre 2016, p. 23.

2. Luca Ronconi, entretien avec Helga Finter, non publié, janvier 2002.

3. Helga Finter, «De l'usage de la voix techniquement assistée sur scène " (conférence au colloque international « Pratiques de la voix sur scène », organisé par Ana Wegner, Chloé Larmet et Marcus Borja, 16-18 novembre 2015, Théâtre Gérard Philipe-Centre dramatique national de Saint-Denis), publié dans A. Wegner, C. Larmet et M. Borja (dir.), Incertains regards, Cahiers dramaturgiques, hors-série 3, Pratiques de la voix sur scène. De l'apprentissage à la performance vocale, Aix-en-Provence, PUP, 2018, p. 123-128.

4. Voir Gustave Flaubert, Madame Bovary. Mours de province, édition établie par Édouard Maynial, Paris, Garnier, 1961, p. 211.

5. Terme créé par Michel Chion, désignant l'instance et la fonction sémiotique d'une voix dont la source est cachée. Voir Michel Chion, La Voix au cinéma, Paris, Cahiers du Cinéma, Éditions de l'Étoile, 1982, p. 28-33.

6. Notons l'emploi du micro pour la voix acousmatique du Commandeur dans le Don Giovanni mis en scène et dirigé par Herbert von Karajan au Festival de Salzbourg en août 1969.

7. Je renvoie ici aux recherches de Michel Poizat. Voir L'Opéra ou le cri de l'ange. Essai sur la jouissance de l'amateur d'opéra, Paris, Métailié, 1986.

8. Voir Marco Beghelli, "Voix et chanteurs dans l'histoire de l'opéra », in Jean-Jacques Nattiez (dir.), Histoires des musiques européennes. Musiques. Une encyclopédie pour le XXI ${ }^{e}$ siècle, vol. 4, Paris, Actes Sud/Cité de la Musique, 2006, p. 522-551.

9. Bertolt Brecht, «Kleines Organon », Ausgewählte Werke in sechs Bänden, vol. 6, Frankfurt/Main, Suhrkamp, 1967, p. 522. (Notre traduction)

10. Voir Alessandro D’Ancona, Origini del teatro, vol. I, Rome [1891], 1971, p. 379-391 ; Helga Finter, «Einsatz des Dramas, Einsatz der Stimme im Theater der italienischen Renaissance», Die soufflierte Stimme, Frankfurt/Main, Peter Lang, 2015, p. 527-540. En France comme dans les pays de langue allemande, cette annonce était effectuée souvent par l'auteur, le souffleur ou le meneur du jeu, et ne comportait donc pas de connotation religieuse.

11. Voir la représentation de L'Annonciation à Florence en 1436, avec la machine du Paradis de Brunelleschi, représentation donnée lors du Concile organisé pour la réunification de l'Église de Rome avec celle de Byzance.

12. Voir Susan Rankin, « Liturgie dramatique et drame liturgique », in J.-J. Nattiez, op. cit., p. 207. 
13. De tels seconds prologues se trouvent par exemple chez Ludovico Dolce. Voir le deuxième dialogue in Leone de' Sommi, Les Quatre Dialogues en matière de représentations théâtrales, traduit de l'italien par Françoise Decroisette, Paris, Rampazzo, 1992.

14. Dans le contexte de la Renaissance et du Baroque la tromba était un instrument en métal d'une longueur de $150 \mathrm{~cm}$ avec un retour d'environ $50 \mathrm{~cm}$. Plus long que la trompette moderne et sans pistons, cet instrument ressemblait plutôt à l'instrument alors appelé «fanfare » en allemand.

15. Voir André Chastel, Marsile Ficin et l'art, Genève, Eugénie Droz, 1954.

16. Ludovico Ariosto, "Prologo alla Cassaria ", in Commedie, La Cassaria I Suppositi, édité par Luigina Stefani, Milan, Mursia, 1997, p. 81, vers 7-8 : «[...] ma con giochi/ si può far una fabula men triste ».

17. Émile Benveniste, « De la subjectivité dans la langue », Problèmes de linguistique générale, Paris, Gallimard, 1966, p. 258-266.

18. Dans l'eucharistie catholique, le vin est blanc pour souligner le mystère de la transsubstantiation en un sang rouge réel. Dans la confirmation protestante, qui reste symbolique, le pasteur emploie du vin rouge.

19. Giorgio Agamben, Le Langage et la mort. Un séminaire sur le lieu de la négativité [Il linguaggio e la morte, Turin, 1986], traduit de l'italien par Marilène Kaiola, Paris, Bourgois, [1991] 1997.

20. Création Le Manège/Théâtre des Amandiers, Nanterre, 2015. Vu à la Filature de Mulhouse en avril 2016.

21. Vu au Maillon, Strasbourg, le 23 novembre 2016.

\section{RÉSUMÉS}

Partant du constat de la sonorisation des voix au théâtre et de l'emploi de voix acousmatiques aujourd'hui, cette contribution questionne la fonction de la vocalité dans la représentation des personnages. À partir d'exemples de sémiotisation du fait vocal, elle propose de considérer celuici comme la structure de base de l'émission de la parole sur la scène européenne et montre sa fonction fondamentale comme scène primitive de la représentation du sujet, laquelle présuppose la reconnaissance de l'autre de la langue et de l'autre du texte.

La qualité spécifique de la vocalité sur scène est ensuite abordée à travers la fonction du rythme et plus largement de la structuration poétique, identifiée comme référent du pouvoir de la parole voire de la souveraineté vocale. L'impact historique et culturel de cette souveraineté est analysé : la parole du théâtre comme le chant de l'opéra tiennent leur pouvoir de procédés employés dans les mystères chrétiens. Cette conception de la représentation effectuée par une voix à la fois personnelle et transcendantale trouve son modèle implicite dans l'eucharistie chrétienne voire catholique. Partagé entre rite chrétien et théâtre antique, le théâtre de la Renaissance s'émancipe par la conceptualisation de la parole poétique, garante d'une voix transcendantale qui présente la représentation. Dès lors, c'est par la voix au théâtre que la représentation rejoue l'origine qui la constitue.

This article focuses on the use of MicroPort and hidden voices in contemporary theatre to examine the function of vocality in the representation of the characters. Using examples of the semiotization of the vocal act, it proposes considering the latter as the fundamental structure of voice emission in European theatre and demonstrates its basic function as the primal scene of 
subject representation, presupposing recognition of the other of language and the other of the text.

The specific quality of vocality on stage is then discussed through the function of rhythm and, more broadly, through poetical structure, identified as a reference for the power of speech, for vocal sovereignty, even. The historical and cultural impact of this sovereignty is explored: voice in opera and theatre derives its power from the legacy of procedures employed in Christian mystery plays. This conception of representation by a voice at once personal and transcendent is implicitly modeled on the Christian, even Catholic, Eucharist. Divided between Christian rite and ancient theatre, the Renaissance theatre broke free by conceptualizing poetic speech, guarantor of a transcendent voice that presents the representation. Thereafter, it was through the theatrical voice that the representation would replay the creation of its origins.

\section{INDEX}

Mots-clés : représentation, Renaissance, voix (du texte), voix (transcendantale), parole (au théâtre), voix d'opéra, technologies sonores

Keywords : representation, Renaissance, voice (textual), voice (transcendental), speech (theatrical), operatic voice, sound technologies

\section{AUTEUR}

\section{HELGA FINTER}

Helga Finter, professeur émérite d'esthétique et d'histoire du théâtre à l'Université Justus-Liebig de Gießen (Allemagne), dont elle a codirigé l'Institut für Angewandte Theaterwissenschaften jusqu'en 2003, est l'auteure d'ouvrages sur la poésie futuriste, les utopies théâtrales de Mallarmé, Jarry, Roussel et Artaud. Elle a dirigé des ouvrages sur le rapport entre le théâtre et les autres arts et sur Georges Bataille. Ses travaux sur la voix et la vocalité au théâtre font internationalement référence, en particulier pour ce qui concerne les usages scéniques des technologies sonores de la fin du XIX ${ }^{e}$ siècle à la période contemporaine. Ses premières recherches ont été publiées à la fin des années 1970, ce qui fait d'elle une pionnière dans le champ. Elle a publié sur le sujet un très grand nombre d'articles et d'études. Les ouvrages récemment parus : Le Corps de l'audible et Die soufflierte Stimme : Text, Theater, Medien (Peter Lang, 2014) présentent l'ensemble d'un parcours où s'articulent esthétique théâtrale, histoire des techniques et psychanalyse.

Parmi ses publications :

El espacio subjetivo. "...el lugar donde el pensamiento ha de encontrar su cuerpo » : Antonin Artaud y la utopia del teatro, Buenos Aires, Ediciones Artes del Sur, 2006.

Das Reale und die (neuen) Bilder (dir.), Peter Lang, 2008.

Medien der Auferstehung (dir.), Peter Lang, 2012.

Le Corps de l'audible. Écrits français sur la voix, 1979-2012, Peter Lang, 2014.

«Sacrifices de la voix », in J.-M. Larrue et M.-M. Mervant-Roux (dir.), Le Son du théâtre (xIX ${ }^{e}$ XXI ${ }^{e}$ siècle), Paris, CNRS Éditions, 2016, p. 391-403.

« De l'usage de la voix techniquement assistée sur scène », in A. Wegner, C. Larmet et M. Borja (dir.), Incertains regards, Cahiers dramaturgiques, hors-série 3, Pratiques de la voix sur scène. De l'apprentissage à la performance vocale, 2018, p. 123-128. 